\title{
NICHE detector and operations
}

\section{Yugo Omura*}

Graduate School of Science, Osaka City University, Osaka, Japan

E-mail: omuraecosmicray-ocu.jp

\section{Kei Nakai}

Graduate School of Science, Osaka City University, Osaka, Japan

E-mail: Skk1159@cosmicray-ocu.ip

\section{Yoshiki Tsunesada}

Graduate School of Science, Osaka City University, Osaka, Japan

E-mail: rtesci.osaka-cu.ac.jp

\section{Douglas R Bergman}

Department of Physics and Astronomy, The University of Utah, Salt Lake City, Utah, USA

E-mail: bergmanephysics.utah.edu

\section{John F Krizmanic}

Universities Space Research Association, Greenbelt, Maryland, USA

E-mail: iohn.f.krizmanicenasa.gov

The Non-Imaging CHErenkov Array (NICHE) is a low energy extension to Telescope Array and TALE using an array of closely spaced $(\sim 200 \mathrm{~m})$ light collectors covering an area of $\sim 2$ square $\mathrm{km}$. It is being deployed in the field of view of TALE and will overlap it in the energy range above $4 \mathrm{PeV}$. Cosmic ray air showers with energies 1-100 PeV will be reconstructed using both the Cherenkov light Lateral Distribution and the Cherenkov time Width Lateral Distribution. These two methods will allow shower energy and $X_{\max }$ to be determined. A prototype of the array, called j-NICHE, has been deployed. Firstly mentioning the performance of detector's components, current status of the detectors and the observations will be presented.

36th International Cosmic Ray Conference -ICRC2019-

July 24th - August 1st, 2019

Madison, WI, U.S.A.

\footnotetext{
* Speaker.
} 


\section{Introduction}

The mass composition of cosmic rays is very important to clarify their origin, because it must be strongly related to their sites of origin, mechanisms of particle accelerations, and propagation from the sources to the Earth. There is a general agreement as regards cosmic ray composition that the fraction of the heavier component increases with energy around the knee region ( $E=10^{15}-10^{16} \mathrm{eV}$, see [四] for review). In air shower experiments, the types of primary nuclei that induce air showers can be inferred from the longitudinal developments of the showers thanks to the differences in the interaction cross-sections with the atmosphere. The results from the previous experiments show that the cosmic-ray mass $\langle\ln A\rangle$ increases with energy indicating a heavy-dominant composition at the knee. This is consistent with the rigidity-dependent stochastic particle acceleration models for cosmic ray sources that predict the maximum reachable energies $E_{\max } \propto Z$. On the other hand, it has been predicted that galactic cosmic ray sources such as supernovae cannot accelerate particles to energies greater than $\sim 10^{18} \mathrm{eV}$, and therefore we conclude that cosmic rays with such high energies are of extra-galactic origin. In this case, protons and other lighter components would be dominant in this higher energy region, since heavier nuclei suffer from photo-disintegration processes by interaction with the cosmic microwave background photons during long distance propagation. In fact, from recent measurements of the cosmic ray composition in the ultra-high energy region $\left(E>10^{18} \mathrm{eV}\right)$ using the air fluorescence detection

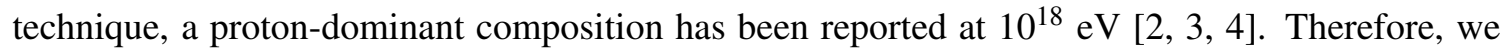
can expect a drastic change in the cosmic-ray mass composition in the energy range of $10^{16}$ to $10^{18}$ $\mathrm{eV}, i$. e. from the heavier galactic components to the lighter extra-galactic components. The aim of the NICHE (Non-Imaging CHErenekov) experiment is to measure the mass composition of cosmic rays in this transient region.

\section{Non-imaging Cherenkov Technique}

Energetic electrons generated in an extended air shower (EAS) produce Cherenkov radiation if they move faster than the speed-of-light in the local medium. The index of refraction in the atmosphere increases with depth, yielding Cherenkov cones from altitudes 8-20 km to overlap in a ring of radius 120-140 $\mathrm{m}$ at the ground. Since the interior of the ring is filled by the portion of the air shower at lowest altitudes, showers that develop deeper in the atmosphere will have a larger interior-to-ring ratio. Electron transverse momentum smears the Cherenkov ring on the ground, but the inside-to-outside ratio remains composition, i.e. $X_{\max }$, dependent.

At a given point on the ground, a counter will observe two components of Cherenkov light: one from the bulk of the shower, where some fraction of the transverse electrons are pointing their Cherenkov cones at the counter; and another part due to the smaller portion of the shower where the shower core Cherenkov cone intercepts the counter. Photons in the former component arrive over a long time span because the measurement samples a large portion of the developing air shower, while the latter component is narrow in time. When there is no dominant core component observed in a counter, the FWHM can be quite wide. Thus the FWHM in time provides a measure of shower development: deeper showers will have more Cherenkov light coming late due to sampling the air shower over a long path length. 
A number of experiments have employed the non-imaging Cherenkov technique (AIROBICC, BLANCA, CACTI, and Tunka) using the Cherenkov Light Distribution (CLD) to measure the CR spectrum, while two experiments (Tunka and BASJE) have employed the Cherenkov Time Width Lateral distribution technique (references in [1], 国]). The innovation of NICHE is to combine these two techniques to construct an array of sufficiently large area to have significant overlap with TALE air-Cherenkov measurements for energies above about $3 \mathrm{PeV}$ and TA/TALE air fluorescence measurements for energies above about $100 \mathrm{PeV}$, leading to a cross-calibration of the FD, IACT and non-imaging CD measurements. It is important to compare energies independently determined from the three types of detectors, the TALE FD, the scintillation counters of the TALE, and the Cherenkov counters of NICHE. Comparing the air shower geometries from these detectors is also intriguing.

\section{NICHE at the TA site}

A Kakenhi Grant by Japan Society for the Promotion of Science (JSPS) was approved in 2014 for four years, and a prototype array of $14 \mathrm{CDs}$ has been developed. This array will be called $\mathrm{j}$-NICHE to distinguish it from other NICHE endeavors. The positions of the deployed j-NICHE counters are shown in the left panel of Fig. W. The detectors are deployed $\sim 800 \mathrm{~m}$ away from the MD site with $100 \mathrm{~m}$ spacing to detect air Cherenkov lights generated by showers with $E \geq 3 \mathrm{PeV}$ together with the MD and the TALE FDs.
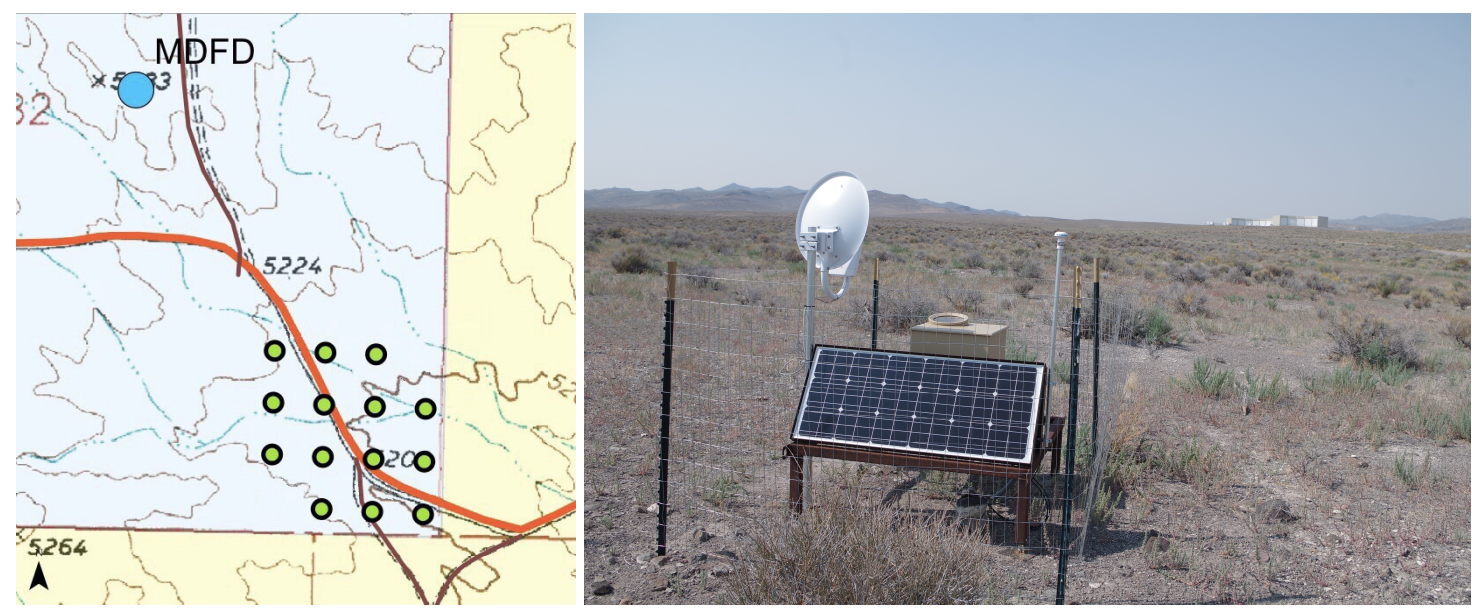

Figure 1: Left: The map around the TA Middle Drum (MD) site. The j-NICHE counters denoted by greens are deployed with $100 \mathrm{~m}$ spacing $\sim 800 \mathrm{~m}$ away from the MD site. Right: A j-NICHE counter in the field with MD-FD behind.

A j-NICHE counter detects Cherenkov light by a 3-inch photomultiplier tube (PMT, Hamamatsu R6233-100) whose the output signal is passed through FADC(200MHz, 12 bits) and stored as a digital in a micro-SD card. A Winston cone of opening half-angle $45^{\circ}$ is attached above PMT to collect more inclined lights. We found the most of rays with $\theta=43^{\circ}$ can not be seen by PMT according to the result of ray-tracing simulation for a NICHE detector using ROBAST[四] (see Fig. (3)). The Winston cones are made by machining a solid aluminum 4-inch dowel at the University of Utah. 
Data acquisition and triggering are handled by a FADC/FPGA unit made by Brains, Inc. in Japan shown in Fig. 凹, that can be connected using SSH via a LAN port as well as receives Bash commands on an incorporated Linux to control a set of components: turning on $\mathrm{HV}$, open/closing a shutter and taking data. Triggering event occurs when 16-points simple moving average gets over an average of the 1024 points without triggering by $\geq 7 \sigma$. Triggered and stored signal has 1024 time points $(\sim 5 \mu \mathrm{s})$ and a timestamp given by a clock count of FADC synchronizing with a PPS signal of GPS, and all the data taken at a night is retrieved via a wire-less communication system just after observation.

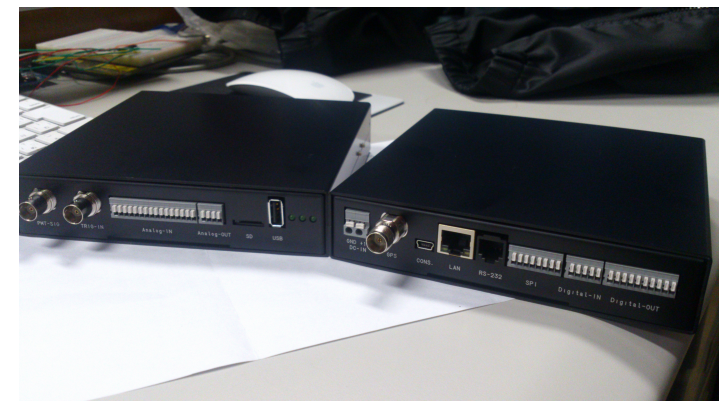

Figure 2: The front and back of units made by Brains, Inc.
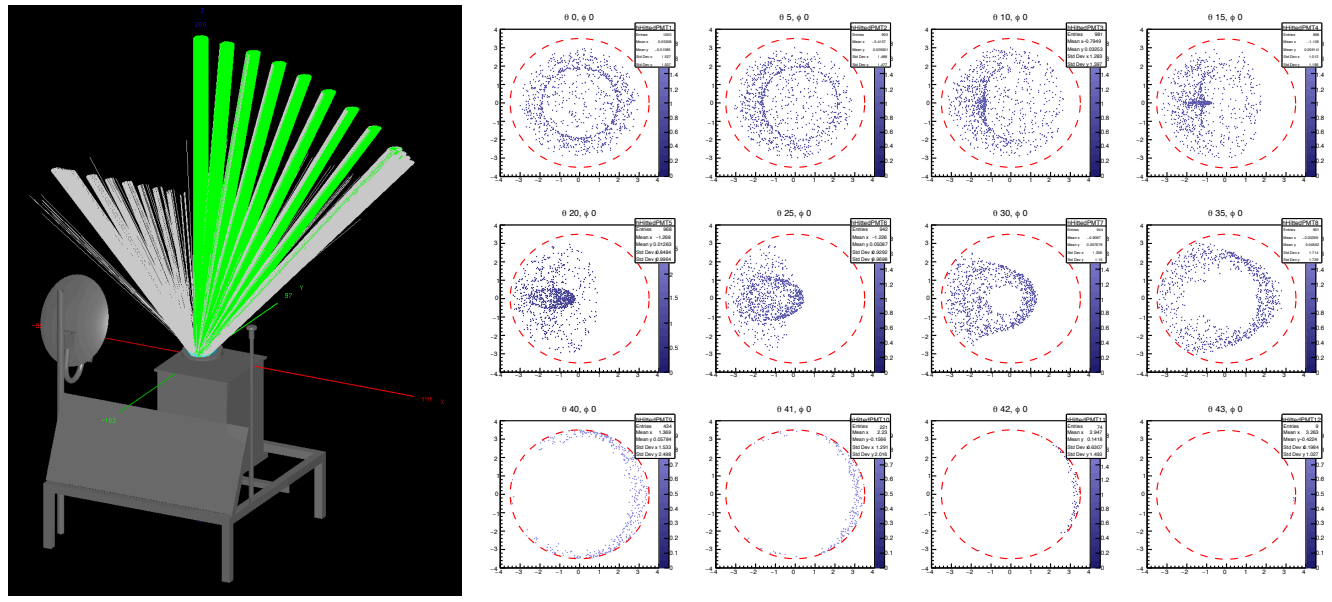

Figure 3: 1,000 rays are generated by changing zenith angle $\theta=0^{\circ}$ to $43^{\circ}$ at azimuthal angle $\phi=0^{\circ}$ and injected randomly in a circle of the upper side of Winston cone. Left: such rays are injected to the detector. Green rays are hits on PMT, otherwise gray ones is not hits because of being blocked by obscurations or going outside through reflected some times in Winston cone. Right: hit points distribution. Dashed red line is a boundary of PMT photocathode area.

The gain curve of every PMT has been measured with YAP $[$ [ $]$ attached on a PMT window. Absolute calibration of the j-NICHE PMTs in terms of a conversion factor from FADC counts to the number of photons were carries out using the CRAYS system, consists of a pulsed nitrogen laser and a chamber filled with a pure gas ( $>99.999 \%, \mathrm{~N}_{2}$ or Ar), which was also used for PMT absolute calibration of TA FDs [Q]. The results is shown in Fig. 田.

The housing for the j-NICHE counters is displayed in Fig. [1. It includes a rotating platter with a hole that serves as a shutter, keeping the PMT and Winston cone safe during the daylight hours. 

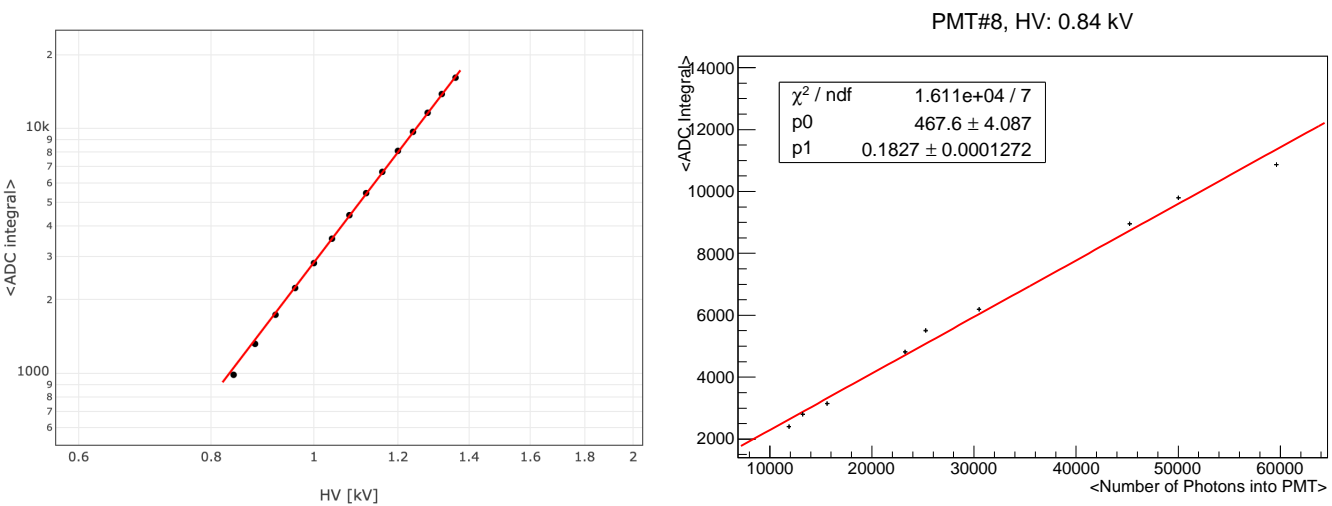

Figure 4: Left: A typical gain curve. Each black point is the average of pedestal-subtracted integral of $\sim 400$ signals by changing HV 0.84 to $1.36 \mathrm{kV}$ with increment by $0.04 \mathrm{kV}$, and a red line is a power-law function fitted all black point shown using a minimizer, MIGRAD based on Davidon-Fletcher-Powell method built in MINUIT[ए]]. Right: CRAYS result. The number of photons into PMT is calculated by using a simple ray-tracing simulation taking into account Rayleigh scattering in the chamber. A red line is a line fit function using MIGRAD and allows us to count the number of photons in real data.

It also houses the batteries, data acquisition and control electronics. The j-NICHE counters have only one PMT installed per counter, but a two-PMT design for future NICHE hybrid array will allow for a local coincidence trigger and thus a lower threshold and/or a lower trigger rate.
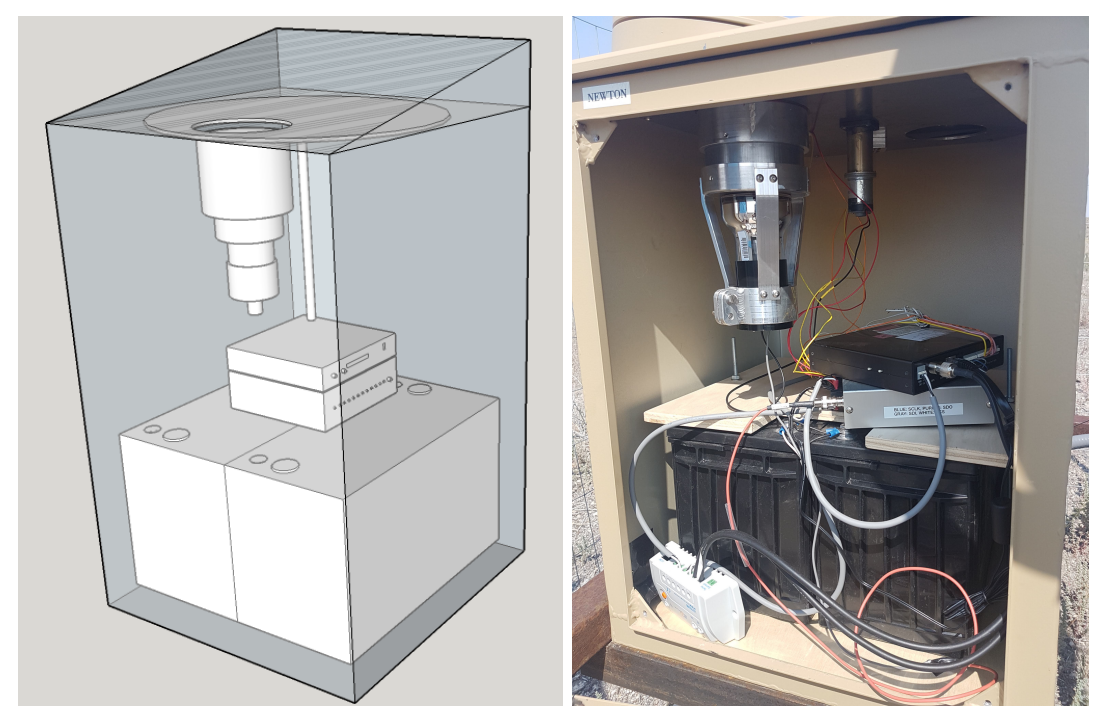

Figure 5: Enclosure of a j-NICHE counter. Left: a CAD image. Right: Actual view of a j-NICHE counter. A PMT, two electronics boxes, batteries, a motor and a charge controller can be seen.

\section{NICHE operation}

NICHE operation is held for a whole dark night from the end of the astronomical twilight to the beginning of the next astronomical twilight, thus carried out at the same time as MD-FD operation. Main PC at MD-FD building connects each detector via wire-less communication using SSH, and 
sends a command and gets a corresponding result using Expect. Preparations of observation begins 30 minutes before the astronomical twilight, connecting and initializing every detectors and turning on HV with a shutter closed. Data taking starts till 15 minutes before the end, after opening a shutter at the beginning, and then data collecting and backup are executed. NICHE observation has regularly started in May 2018 when 10 counters were deployed then, but all the counter is done in Sep 2018. Total observation time is 103 days/625 hours and the number of coincidence events with over 4 detectors is 110,768 until June 2019. A typical trigger rates and a rate of coincidence events in 31 Mar 2019 are shown in Fig. 6.
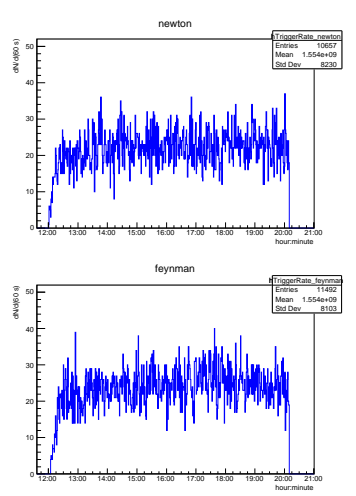
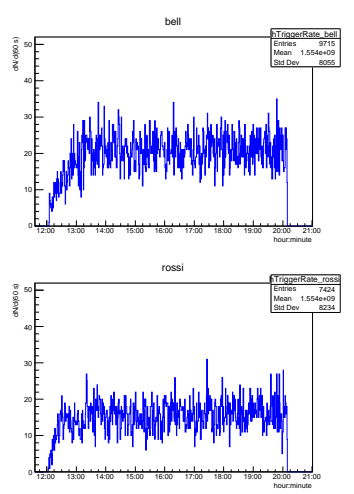

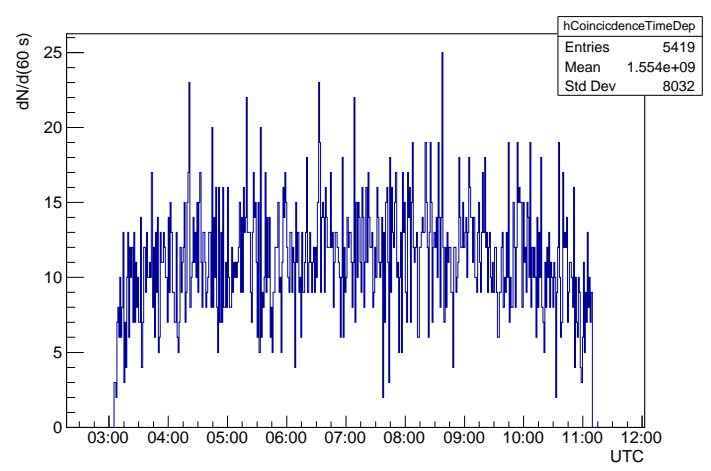

Figure 6: Left: Typical trigger rates per a minute. Right: A rate of coincidence events $(\geq 4)$ per a minute in right. In 31 Mar 2019.

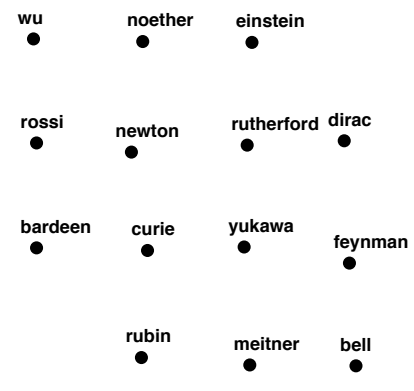

Figure 7: The name of every detectors.

\section{Summary}

NICHE experiment aims to measure the cosmic-ray composition in $10^{16}$ to $10^{18} \mathrm{eV}$ by detecting Cherenkov lights generated by air-shower with 14 detectors placed in a grid and spaced 100 $\mathrm{m}$ apart. Each detector has mainly a single PMT and a FADC and a Winston cone attached above PMT makes it possible to detect lights with zenith angle $<\sim 43^{\circ}$. All NICHE detector has been deployed and taken data since May 2018. 


\section{References}

[1] Karl-Heinz Kampert, Michael Unger, Measurements of the cosmic ray composition with air shower experiments, Astropart. Phys. 35 (2012) 660

[2] R.U. Abbasi et al. (The Hiresolution Fly's Eye Collaboration), Phys. Rev. Lett., 104, 161101-1-5 (2010)

[3] J. Abraham et al. (Pierre Auger Collaboration), Phys. Rev. Lett., 104, 091101-1-7 (2010)

[4] R.U. Abbasi et al. (The Telescope Array Collaboration), Astropart. Phys., 64, 49-62 (2015)

[5] J. Krizmanic, D. Bergman, P. Sokolsky, The Non-Imaging CHErenkov Array (NICHE): A TA/TALE Extension to Measure the Flux and Composition of Very-High Energy Cosmic Rays, Proceedings of the 33rd ICRC (Rio de Janeiro), (2013) 0365.

[6] D. Bergman, J. Krizmanic, NICHE: The non-imaging Cherenkov array, AIP Conf. Proceed. 1516 (2013) 282.

[7] Akira Okumura, Koji Noda, Cameron Rulten, ROBAST: Development of a ROOT-Based Ray-Tracing Library for Cosmic-Ray Telescopes and its Applications in the Cherenkov Telescope Array, Astropart. Phys. 76 (2016) 38

[8] Kobayashi M, Shinkawa T, Sato T et al., Nucl. Instr. and Meth. A 337, 355 (1994).

[9] S. Kawana, M. Fukushima, Y. Tsunesada et al., Nucl. Instr. Meth. A, 681, 68-77 (2012)

[10] F.James and M.Roos, Comput.Phys.Commun., 10 (1975) 343-367 